\title{
Radiological and neurophysiological changes in Japanese encephalitis
}

\author{
U K Misra, J Kalita, S K Jain, A Mathur
}

\begin{abstract}
Six patients with Japanese encephalitis, four males and two females whose age ranged between 2 and 47 years, were subjected to neurophysiological and radiological studies. An EEG in five of the patients showed diffuse delta wave activity and one had an alpha coma. Delta activity seems to be due to thalamic involvement, which was seen on CT of two and MRI of all the patients. The thalamic lesions were characteristically bilateral and were haemorragic in five. Changes on MRI included abnormalities of the brainstem in three and the basal ganglia and spinal cord in one patient each. Lower motor neuron signs were present in three patients but abnormal MRI signals in the spinal cord were present in only one out of three patients in whom spinal MRI was carried out. Central motor conduction time in the upper limb was prolonged in three patients (five sides) and in the lower limbs in one (both sides), which was consistent with involvement of the cerebral cortex, thalamus, brainstem, and spinal cord. Changes in MRI and EEG in the acute stage may provide early diagnostic clues in patients with Japanese encephalitis.
\end{abstract}

(F Neurol Neurosurg Psychiatry 1994;57:1484-1487)

Sanjay Gandhi Post Graduate Institute of Medical Sciences, Post Box 375, Lucknow, India Department of Neurology U K Misra

J Kalita

Department of Radiology

S K Jain

Department of

Virology, KG Medical College, Lucknow, India

A Mathur

Correspondence to:

Professor U K Misra, Pepartment of Neurology, Sanjay Gandhi Post Sanjay Gandhi Post Mraduate Institute of Medical Sciences, Raebare India.

Received 15 November 1993 and in final revised form 10 June 1994 Accepted 22 June 1994
Epidemics of Japanese encephalitis are prevalent in the Indian subcontinent. Japanese encephalitis is common in the rainy season, from June to September. Clinical and epidemiological studies on Japanese encephalitis have been reported. ${ }^{12}$ The diagnosis of Japanese encephalitis depends on the demonstration of rising titres of antibodies in serum samples, which delays the diagnosis by a few weeks. Differentiation of Japanese encephalitis from other types of encephalitis, especially herpes simplex, is important. Little attention has been paid to the changes in EEG, CT, and MRI in Japanese encephalitis. ${ }^{3-5}$ Careful analysis of clinical, radiological, and electrophysiological changes may improve the understanding of Japanese encephalitis and help in early diagnosis.

\section{Patients and methods}

Six patients were confirmed to have Japanese encephalitis by positive haemagglutination inhibition tests for IgM antibodies to Japanese encephalitis virus. ${ }^{6}$ A fourfold or higher rise was considered diagnostic of Japanese encephalitis infection. Neurological assessment included the Glasgow coma scale, muscle power, reflexes, and sensations. Detailed evaluation of autonomic function (blood pressure response to change of posture and cold immersion, handgrip, and RR interval variation on respiration) was performed in the convalescent phase as and when the patient was sufficiently cooperative. Dysautonomia was diagnosed if two or more of these tests were abnormal. An EEG was recorded with a 10 channel machine employing a 10-20 system of electrode placement. Median somatosensory evoked potentials were recorded by stimulating the median nerve at the wrist, recording from Erb's point and the contralateral parietal cortex with a midfrontal reference. Tibial somatosensory evoked potentials were obtained by stimulating the tibial nerve at the ankle and recording from the spinous process of $\mathrm{L} 1$ vertebra referred to $\mathrm{L} 3$ and from $\mathrm{Cz}$ with a mid-frontal reference. Central sensory conduction time (CSCT) was calculated by subtracting the Erb's point or L1 latency from the parietal cortex (N19) or vertex (P40) latency respectively. ${ }^{7}$ Central motor conduction time (CMCT) was studied by transcranial electrical stimulation. The CMCT to the abductor digiti minimi was calculated by subtracting the latency on $\mathrm{C} 7 \mathrm{stim}$ ulation from that on cortical stimulation; similarly CMCT to the tibialis anterior was calculated by subtracting the latency on L1 stimulation from that on $\mathrm{Cz}$ stimulation. ${ }^{8}$ Computed tomography (Hitachi W400, Japan) was performed taking $5 \mathrm{~mm}$ plain and contrast enhanced axial sections, parallel to the orbitomeatal line. Brain MRI was carried out on a $1.5 \mathrm{~T}$ scanner (Magnetom $22614 \mathrm{SP}$, Siemens AG, Germany) with T1, T2, and proton density weighted spin echo sequences. Spinal MRI was also performed. Gadolinium enhancement was not carried out. Mental state was assessed with the mini mental scale during the follow up. The clinical, EEG, and evoked potential studies were repeated after one and three months. The recovery of the patients were classified into poor, partial, or complete recovery at three months. Poor recovery was defined as bedridden, partial recovery as dependence for activities of daily life, and good recovery as complete independence for such activities.

Results

All the patients had the typical clinical picture 
Table 1 Clinical features of fapanese encephalitis $(n=6)$

\begin{tabular}{lll}
\hline Clinical features & 1st week & 12th week \\
\hline Encephalitic syndrome & 6 & - \\
Decerebration/decortication & $4 / 2$ & - \\
Hemiparesis/tetraparesis & $3 / 3$ & $1 / 1$ \\
Autonomic dysfunction & - & 2 \\
Extrapyramidal signs: & 1 & 3 \\
Rigidity & 1 & 1 \\
Tremor & 0 & 3 \\
Hypokinesia & 0 & 1 \\
Dystonia & 0 & 2 \\
Cerebellar signs & 0 & 2 \\
Focal wasting & 0 & 3 \\
Sensory impairment & $?$ & 0 \\
\hline
\end{tabular}

comprising fever, headache, vomiting, and altered sensorium. Meningeal signs were present in two patients. One child had partial motor seizures. All the patients had varying degrees of coma; the Glasgow coma scale ranged between 5 and 7 . Decerebration was present in four and decortication in two patients in the acute stage. Hemiparesis and tetraparesis were present in three patients each. On admission the tendon jerks were brisk in all the patients; the biceps reflex was absent in two and the ankle reflex was absent in one. On follow up the absent biceps reflex had returned to normal after one month in one patient (No 1) but areflexia of the biceps and ankle persisted in the other (No 6). As consciousness improved, mutism and staccato speech were noted in one patient each. Extrapyramidal signs were present in three patients, and included mask-like facies, hypokinesia, tremor, rigidity, and dystonia. These changes were noted during the recovery period as the patients improved from a deep coma. The extrapyramidal signs persisted but were less prominant at the three month follow up. Three patients had fully recovered after three months. Table 1 shows the clinical features in the acute stage and after three months. Autonomic functions were evaluated in all the patients except one who was only 22 months old. Autonomic abnormalities present in patients 1 and 4 included lack of $R R$ variation during respiration, and no rise in blood pressure on isometric contraction and cold immersion. Patient No 1 had giddiness on standing and more severe postural hypotension $(40 / 10 \mathrm{~mm} \mathrm{Hg})$ than patient No 4 who did not have any symptoms related to dysautonomia. These abnormalities were still present at three months.

An EEG in five out of six patients showed bilateral delta wave slowing. One patient who had partial motor seizures had spikes on the left side and voltage asymmetry that correlated with a frontoparietotemporal lesion on MRI. The EEG had returned to normal in four patients after three months. In a patient with a deep coma, an unresponsive alpha-like activity of $20-30 \mu \mathrm{V}$ and $8-9 \mathrm{~Hz}$ was present that was pronounced in the posterior and central regions. The amplitude and frequency did not change on painful stimuli or passive eye opening. A moderate amount of $50-100 \mu \mathrm{V}$, $3 \mathrm{~Hz}$ activity was also present bifrontally. This patient improved appreciably; after eight months of her illness she had a cerebellar dysarthria and limb ataxia, although she could walk unaided. Her mini mental scale score was 30 but EEG did not show any change at follow up. In this patient (No 4) MRI on the 30th day of illness while she was still comatose showed bilateral thalamic and pontine lesions.

Motor evoked potentials were studied in four patients (eight sides); CMCT-ADM was prolonged in three (five sides) and ranged between 8.2 and $11.4 \mathrm{~ms}$. The CMCT-TA was prolonged bilaterally in one patient (18.4 $\mathrm{ms}$ on the right and $19.6 \mathrm{~ms}$ on the left side). Central motor conduction abnormality correlated with abnormality on spinal MRI in one patient only (No 1). Median and tibial somatosensory evoked potentials were normal in all the patients except one, in whom a left somatosensory evoked potential was not recordable. In this patient (No 2) spinal MRI was normal. Ulnar motor and sensory nerve conduction and sural sensory conduction studies were normal. Peroneal motor conductions were unrecordable in one patient bilaterally, because of pronounced atrophy of the extensor digitorum brevis muscles; an EMG showed fibrillation and no motor unit potential was recordable from either side. An EMG of other wasted muscles-namely, first dorsal interosseus, brachioradialis, supinator, and biceps showed pronounced reduction in recruitment.

Computed tomography was performed on five patients. It showed non-enhancing low

Table 2 Distribution of MRI changes in patients with fapanese encephalitis

\begin{tabular}{|c|c|c|c|c|c|c|c|c|c|c|c|c|}
\hline & \multicolumn{12}{|c|}{ Patients number } \\
\hline & \multicolumn{2}{|l|}{1} & \multicolumn{2}{|l|}{2} & \multicolumn{2}{|l|}{3} & \multicolumn{2}{|l|}{4} & \multicolumn{2}{|l|}{5} & \multicolumn{2}{|l|}{6} \\
\hline & $R$ & $L$ & $R$ & $L$ & $R$ & $L$ & $R$ & $L$ & $R$ & $L$ & $R$ & $L$ \\
\hline \multicolumn{13}{|l|}{ Site: } \\
\hline Cortex & - & - & + & + & - & + & - & - & + & + & + & - \\
\hline Cerebellum & - & - & + & + & + & + & - & - & - & + & - & - \\
\hline & + & + & + & + & + & + & + & + & + & + & + & + \\
\hline Basal ganglia & - & - & - & - & - & - & - & - & - & - & + & + \\
\hline Pons & $\overline{-}$ & - & + & + & - & - & + & + & - & - & - & - \\
\hline $\begin{array}{l}\text { Midbrain } \\
\text { Spinal cord }\end{array}$ & \multicolumn{2}{|c|}{+} & \multicolumn{2}{|c|}{-} & \multicolumn{2}{|c|}{-} & \multicolumn{2}{|c|}{ ND } & & \\
\hline \multicolumn{3}{|c|}{$\begin{array}{l}\text { Nature of thalamic lesion } \\
\text { (T1, T2) }\end{array}$} & \multicolumn{2}{|c|}{$\mathrm{H}$} & \multicolumn{2}{|c|}{$\mathbf{H}$} & \multicolumn{2}{|c|}{$\mathrm{H}$} & \multicolumn{2}{|c|}{$\mathrm{H}$} & \multicolumn{2}{|c|}{ NH } \\
\hline $\begin{array}{l}\text { Day of MRI } \\
\text { Recovery }\end{array}$ & \multicolumn{2}{|c|}{$\begin{array}{c}10 \\
\text { Complete }\end{array}$} & \multicolumn{2}{|c|}{$\begin{array}{c}20 \\
\text { Complete }\end{array}$} & \multicolumn{2}{|c|}{$\begin{array}{c}15 \\
\text { Partial }\end{array}$} & \multicolumn{2}{|c|}{$\begin{array}{c}30 \\
\text { Partial }\end{array}$} & \multicolumn{2}{|c|}{$\begin{array}{c}60 \\
\text { Complete }\end{array}$} & \multicolumn{2}{|c|}{$\begin{array}{c}60 \\
\text { Poor }\end{array}$} \\
\hline
\end{tabular}

$\mathrm{H}=$ haemorrhagic; $\mathrm{NH}=$ non-haemorrhagic; $\mathrm{ND}=$ not done; $\mathrm{R}=$ right; $\mathrm{L}=$ left. 
Figure 1 Axial MRI on (A) $T 1$ and (B) $T 2$ sequence showing bilateral thalamic lesions; patient No 4.

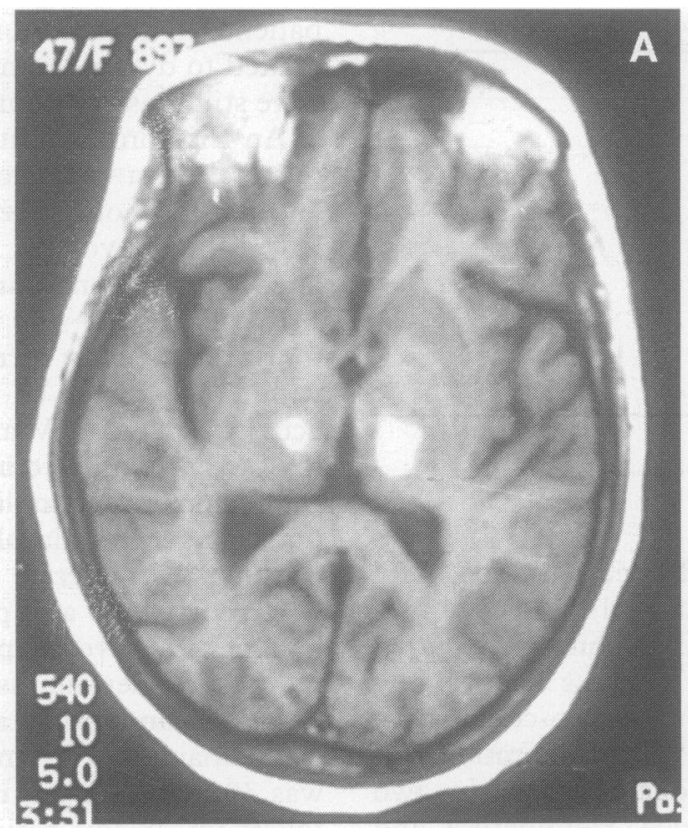

density areas in the thalamus and pons in two patients and a low density area witk. haemorrhage in the thalamus in one. Varying amounts of white matter oedema were present in all five patients. Cranial MRI was done in all six and spinal MRI in three patients. Table 2 shows the distribution of MRI lesions in the CNS. Magnetic resonance imaging was performed within one month in three patients and after one month in the other three. The thalamic lesions showed signal changes suggestive of subacute haemorrhage in these patients (fig 1). In other regions the changes on $T 1$ and $T 2$ weighted images varied from non-haemorrhagic (hypointense in $\mathrm{T} 1$ and hyperintense in T2) to haemorrhagic areas.

Cerebrospinal fluid was abnormal in all the patients but one. Mean CSF protein was

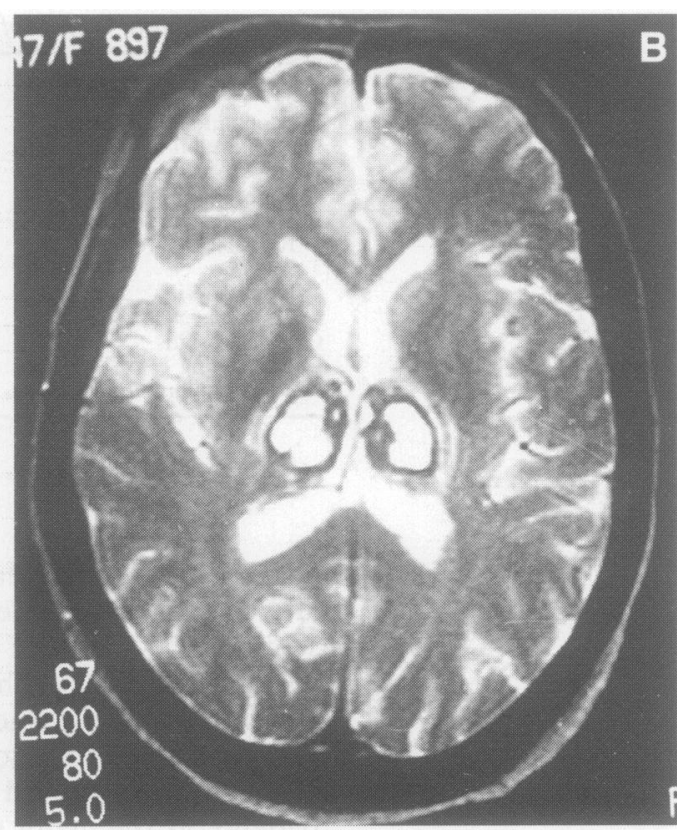

$162 \cdot 4$ (range 34-471) $\mathrm{mg} / \mathrm{dl}$, cell count was 19 (range 3-60) $\mathrm{mm}^{3}$. The cells were mainly lymphocytes. The CSF was negative for bacteria and fungi both by direct staining and culture. All the patients had specific IgM antibodies against Japanese encephalitis virus in acute phase serum samples. Antibody titres, as measured by haemagglutination inhibition, in the initial serum samples from four patients were 1:40 and in two they were $1: 20$. All patients showed a fourfold or greater rise $(1: 80$ to $1: 320)$ in convalescent serum samples.

\section{Discussion}

The characteristic MRI finding in Japanese encephalitis is bilateral haemorrhagic lesions
Figure 2 EEG in a patient with fapanese encephalitis, on admission, at 60 days and at 90 days showing progressive improvement.

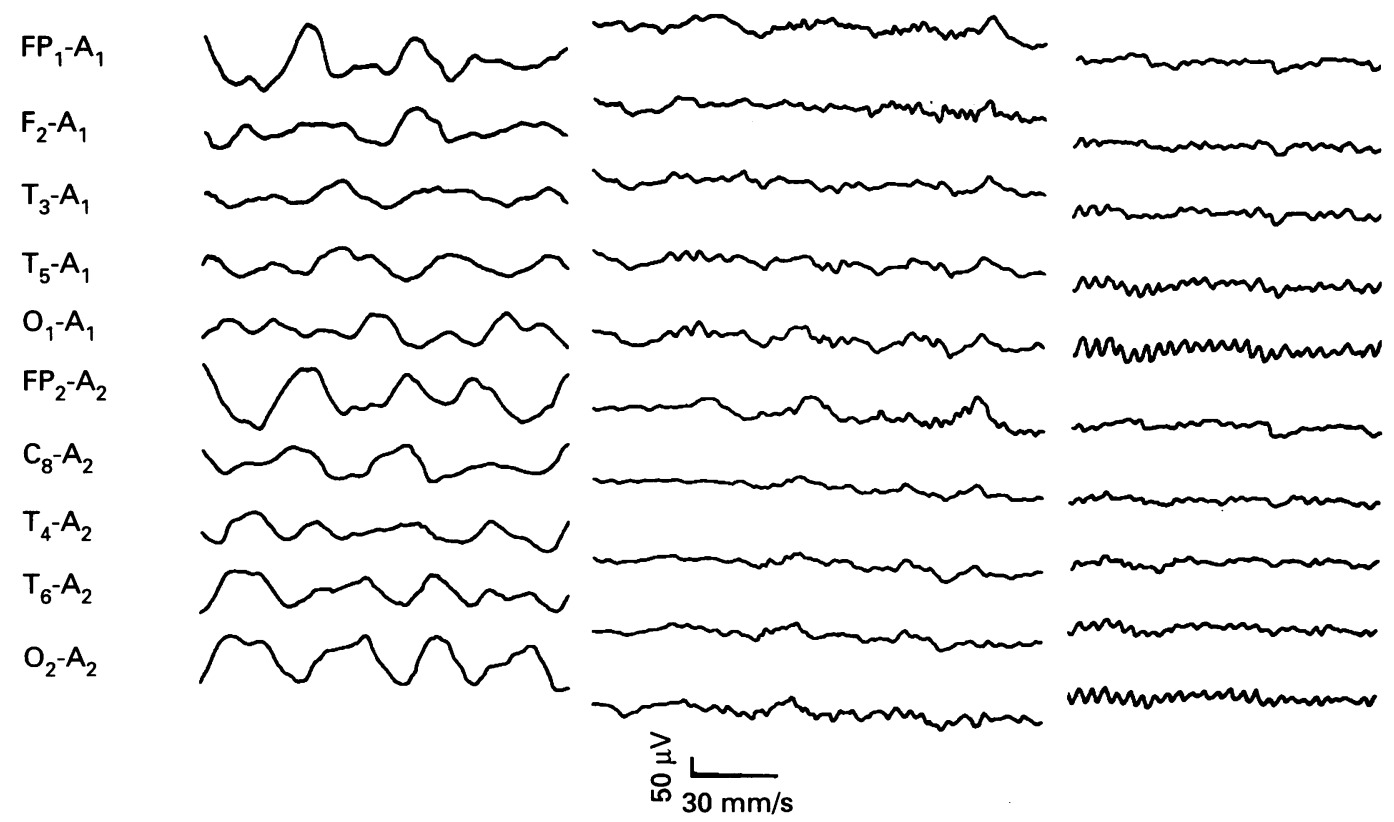

Day 2 Mini-mental scale 
in the thalami. There are only two reports of MRI changes in Japanese encephalitis. In the first the MRI was performed on a $0.5 \mathrm{~T}$ scanner, and the changes included hyperintense lesions in T2 in the thalami. Some of these patients, however, had previous cerebral infarctions, which render the interpretation of the MRI difficult. ${ }^{4}$ In the other study the hyperintense lesions in T2 were reported after five months to three years and persisted even after three years. ${ }^{9}$ The MRI findings in our study are consistent with the histopathological changes in Japanese encephalitis, which include a polymorphonuclear or lymphocytic inflammatory response with neuronophagia, glial proliferation, and focal haemorrhagic areas. These changes are mainly found in the cerebral cortex, basal ganglia, brainstem, cerebellum, and spinal cord.

Japanese encephalitis is an encephalomyelitis. ${ }^{1011}$ In three of our patients focal wasting and EMG changes were suggestive of anterior horn cell involvement but abnormal MRI signals in the spinal cord were found in one patient only. Spinal cord involvement in Japanese encephalitis, however, is well known $^{1011}$ but MRI findings have not been reported so far. Changes in MRI in viral myelitis are similar to those in multiple sclerosis, and spinal MRI is positive in $75 \%$ to $86 \%$ of the patients with clinically suspected multiple sclerosis. ${ }^{12}$ It is possible that gadolinium enhancement could have shown a higher frequency of spinal cord involvement in our study.

The EEG in our patients showed a constant pattern of diffuse delta activity, which seems to be due to bilateral thalamic involvement. Thalamic lesions produce delta waves of varying degree and extent encompassing the whole ipsilateral hemisphere. Delta waves can be generated by lesions of the thalamus in cats. ${ }^{13}$ In our patients the improvement in the EEG correlated with clinical recovery (fig 2). Similar EEG changes in the acute stage have been reported by other investigators. ${ }^{35}$ Spikes and seizure discharges are rare and a burst suppression pattern has been reported in deeply comatose patients. Although EEG reflects the severity of brain damage it is not a good predictor of the outcome. ${ }^{3}$ Association of alpha coma with Japanese encephalitis has not been reported earlier. Our patient with alpha coma improved, contrary to its usually poor prognosis. The involvement of the brainstem at or below the level of the pontomesencephalic junction is responsible for alpha coma. In some patients the lesions have extended rostrally into the midbrain and thalamus, but in these patients a part of the brainstem tegmentum with its ascending pathways to the cortex seems to be spared. These are sufficient to maintain the alpha pattern but insufficient for full consciousness. ${ }^{14}$

Sensory impairment in Japanese encephalitis seems to be less prominent and less frequent compared with motor deficits. Sensory examination was normal in all the patients and somatosensory evoked potentials were also normal in all except one side. In another study the sensory abnormality was present in two out of 68 patients. ${ }^{15}$ Central motor conduction was abnormal in the upper limb in five sides, suggesting the impairment of large diameter fast conducting motor pathways. The prolongation of CMCT is a feature of subcortical damage whereas inexcitability of central motor pathways is found in both cortical and subcortical involvement. ${ }^{7}$ In our study the high frequency of prolonged CMCT may be due to widespread involvement of white matter, thalamus, brainstem, and spinal cord. Lower motor neuron involvement resulting in wasting and loss of reflexes seems to be due to anterior horn cell defects, because of normal nerve conduction studies and neurogenic changes in EMG. The common occurrence of extrapyramidal dysfunction in Japanese encephalitis is attributed to involvement of the substantia nigra and basal ganglia. ${ }^{15}$ In our study only one patient had appreciable and another minimal signal alteration in the midbrain. One patient with severe rigidity tremor and mutism had no midbrain involvement on MRI. These features in the absence of a midbrain lesion may be attributed to bilateral thalamic disruption, as the thalamus is the final common output for the extrapyramidal system. ${ }^{16}$

Bilateral thalamic involvement, especially haemorrhagic damage, seems to be the most common finding on MRI in Japanese encephalitis. In a patient with clinically suspected encephalitis, specially in an endemic area, characteristic MRI and EEG changes may provide important diagnostic clues before serological confirmation.

1 Lincoln AF, Sivertson DE. Acute phase of Japanese B encephalitis. Two hundred and one cases in American soldiers in Korea 1950. $\mathscr{f} A M A$ 1950;150:268-73.

2 Nag D, Misra UK, Shukla R, Kar AM, Chaturvedi UC, Mathur A. Some observations on Japanese encephalitis (JE) in Uttar Pradesh. F Assoc Physicians India 1981;29: 293-8.

3 Gourie Devi M, Deshpande DH. Japanese encephalitis. In: Prasad LS, Kulezyekills T, eds. Pediatric problems. In: Prasad LS, Kulezyekills T, eds. Pediat

4 Shoji H, Hiraki U, Kuwasaki N, Toyomasu T, Kaji M, Okudera $T$. Japanese encephalitis in the Kurume region of Japan. CT and MRI findings. $f$ Neurol 1989;236:255-9.

5 Shoji S, Murakami T, Murai I, Kida H, Sato Y, Kojima K, Abe T, Okudera T. Follow-up study by CT and MRI in three cases of Japanese encephalitis. Neuroradiology 1990;32:215-19.

6 Mathur A, Arora KL, Rawat S, Chaturvedi UC. Persistance, latency and reactivation of Japanese encephalitis virus infection in mice. $f$ Gen Virol 1986;67:381-5.

7 Macdonell RAL, Donnan GA, Bladin RF. A comparison of somatosensory evoked and motor evoked potential in of somatosensory evoked and motor

8 Misra UK, Sharma VP. Central and peripheral conduction studies in neurolathyrism. $\mathcal{F}$ Neurol Neurosurg Psychiatry studies in neuro

9 Joshita Y, Suzuki S, Oowada N, Mizuno Y, Yoshida M. A case of Japanese encephalitis manifesting remarkable changes in MRI, CT and EEG. Neurol Med Chir (Tokyo) 1988,29:409-15.

10 Zimmerman HM. Pathology of Japanese encephalitis. $A m \mathcal{F}$ Pathol 1946,22:965-91.

11 Kumar R, Agarwal SP, Waklu I, Misra PK. Japanese encephalitis - an encephalomyelitis. Indian Pediatri 1991;23:1525-33.

12 Greenberg JO. Neuroimaging of spinal cord. In: Woolsey RM, Young RR, eds. Neurology clinics-disorders of spinal cord, Vol 9. Philadelphia: WB Saunders Company, 1991:679-704

13 Gloor P, Ball G, Scheul N. Brain lesions that produce delta waves in EEG. Neurology 1977;27:326-33.

14 Westmoreland BF, Klass DW, Sharburgh FW, Reagon TJ. Alpha coma: electroencephalographic clinical, pathoAlpha coma: electroencephalographic clinical, pathologic and etiop

15 Richter RW, Shinojyo S. Neurologic sequelae of Japanese B encephalitis. Neurology 1961;11:553-9.

6 Alexander GE, Delong MR. Central mechanisms of initiation and control of movement. In: Asbury AK, McKhann GM, McDonald WI, eds. Diseases of the nervous system. Clinical neurobiology Vol 1. Philadelphia: WB Saunders Company, 1992:285-308. 